Some factors - such as birth weight, maternal anemia, preeclampsia, and existence of congenital diseases-have been clearly defined as prognostic for surviving birth. Pregnancy at high altitude is associated with a reduction in birth weight of approximately $100 \mathrm{~g}$ per $1000 \mathrm{~m}$ of ascent. Low birth weight affects mostly children from mothers without high altitude ancestry, but not native babies, and evidence of endoplasmatic reticulum stress and protein synthesis inhibition in the placenta of nonnative women at high altitude have been found. Children from natives may be protected by the augmented uterine artery flow described for this group. Also, there is the possibility that elevated enzymatic antioxidant activity contributes to the Andean protection against altitude-associated small for gestational age infants. Hemoglobin concentrations in mothers at high altitude are well studied. When appropriate adjustments were used, maternal anemia was associated with lower infant Apgar scores at both 1 minute and 5 minutes after birth, as well as complication of labor, lower gestational age at birth, and higher parity. Little is reported about congenital heart diseases, their natural history, especially regarding pulmonary hypertension, and the unique physiology of indigenous high altitude residents in this context.

Our investigation team has been working on this last topic since 2003. We report the results of 4000 echocardiographic studies performed in 3500 patients born at $3600 \mathrm{~m}$ in La Paz, Bolivia, not older than 3 months. The patient group was selected by pediatricians and referred because of heart murmur, cyanosis, syndromal fenotype, heart failure, oxygen dependency, and enhanced cardiac size in the Rx. Of these, $20.1 \%$ of the children showed no abnormal finding, $18.1 \%$ showed pulmonary hypertension (defined as systolic pulmonary pressure of more than $40 \mathrm{~mm} \mathrm{Hg}$ or more than two thirds from the systemic pressure) in the absence of congenital heart disease, $14.6 \%$ showed ventricular septal defect, $20 \%$ presented with patent ductus arteriosus, $14.2 \%$ atrial septal defect, $5.1 \%$ tricuspidal atresia, 2.6\% complete atrioventricular channel, $2.5 \%$ tetralogy of Fallot, $2.5 \%$ Shone complex, and $0.4 \%$ others.

We assume that the high incidence of pulmonary hypertension in this group, with oxygen dependency and right ventricle hypertrophy for weeks, is due to a prolonged postnatal adaptation period. The most important reason for this phenomenon is the late remodeling of the pulmonary artery branches, which implies the persistence of a thick musculary media. The persistent elevation of the pulmonary pressure and hypoxia are predisposing factors to patency of the ductus arteriosus. Rightsided congenital heart diseases like tricuspid atresia are 3 times more frequent in our patient cohort than at sea level; being leftsided significantly lowers their incidence. Environmental and genetic circumstances may both play a role in the pathogenesis of congenital heart disease at high altitude.

Alexandra Heath, MD, PhD La Paz, Bolivia

\section{How the Central Nervous System Copes With Hypoxic Challenges}

All mammals developed effective strategies to cope with reduced oxygen availability or other metabolic, environmental, and behavioral challenges. An important prerequisite for survival is the necessity to maintain functional integrity during times of extreme challenges. Mammals accomplish this task through a highly coordinated, systems- and cellular-level reconfiguration involving the partial shutdown of some but not all organs. This reconfiguration is controlled through a similarly complex reconfiguration at the cellular and network level within the central nervous system. During hypoxic exposure, for example, active cellular networks must continue to control vital functions, such as cardiorespiratory coupling. In this lecture, I will discuss the ability of the cardiorespiratory network to assume multiple states that adapt the organism to continuously changing external and internal conditions.

Jan Marino-Ramirez, $\mathrm{PhD}$

Seattle, WA, USA

\section{Cardiorespiratory Coupling in Health and Dis- ease: From Bench and Wilderness to the Bedside}

The heart beating and breathing are intricately linked, both functionally as well as anatomically. Within the central nervous system, we find highly overlapping brainstem networks that control these autonomic physiologies. Both of these activities are essential for survival, and the cardiorespiratory coupling has many potential benefits, creating synergies that promote healthy physiology. When this coupling deteriorates, autonomic dysautonomia ensues. In this lecture, I will discuss the neuronal control of this coupling in health and disease and describe how hypoxia can significantly alter these interactions as well as the modulatory control. I will describe how reactive oxygen species act on the neuronal network that provides the biological basis of respiratory sinus arrhythmia. I will also discuss how intermittent hypoxia alters the modulatory state of neuronal networks that ultimately drives these networks into instability.

Jan Marino-Ramirez, $\mathrm{PhD}$

Seattle, WA, USA

\section{Hypoxia and Cancer}

Cancer is a heterogeneous group of disorders linked by unregulated growth of abnormal cells characterized by several genetic aberrations. Hypoxia is a phenomenologic concept produced by lack of oxygen. It depends on the demand of the cells and tissues of the organ. Approximately $90 \%$ of cancers are solid tumors; the rest are hematologic cancers. Hypoxia seems to play a major role in most solid tumors in their characteristic behaviors.

A cancer cell is the most evolved, rapidly adapting moiety, probably in the universe. The evolutionary abilities of the cancer cell program it to continue to grow and multiply even under adverse conditions. Hypoxia occurs in a tumor even when it is as small as $5 \mathrm{~mm}$. The genetic alterations in the hypoxic cell are several. Equally important are the production and release of cytokines such as vascular endothelial growth factor (VEGF), hypoxia-inducible factor-1 (HIF-1), and mutation in the p53 